\title{
Millijoule-level sub-cycle pulses from two channels of a parallel parametric waveform synthesizer
}

\author{
Fabian Scheiba ${ }^{1,2, *}$, Giulio Maria Rossi $^{1,2}$, Roland E. Mainz ${ }^{1,2}$, Yudong Yang ${ }^{1,2}$, Giovanni \\ $\mathrm{Cirmi}^{1,2}$, and Franz X. Kärtner ${ }^{1,2}$ \\ ${ }^{1}$ Center for Free-Electron Laser Science, Notkestrasse 85, 22607 Hamburg, Germany \\ ${ }^{2}$ Physics Department and The Hamburg Centre for Ultrafast Imaging, University of Hamburg, Luruper \\ Chaussee 149, 22761 Hamburg, Germany
}

\begin{abstract}
We report on an optical synthesis of two compressed channels from our parametric waveform synthesizer, leading to a $0.6 \mathrm{~mJ} 3.4 \mathrm{fs}$ pulse ( $3.2 \mathrm{fs}$ transform limited) with a central wavelength of $1.8 \mu \mathrm{m}$, corresponding to 0.6 optical cycles.
\end{abstract}

\section{Introduction}

Laser sources capable of generating few cycle optical pulses allow to observe light-triggered dynamics with femtosecond resolution (ultrafast spectroscopy), as well as to drive strong field processes such as High Harmonic Generation (HHG), that opens up the possibility to observe even faster dynamics, happening over attosecond (as) time scales. While Ti:Sa lasers support $\sim 20$ fs pulses with multi-mJ pulse energy at $\sim \mathrm{kHz}$ repetition rates already, many labs employ the spectral broadening in hollow core fibres (HCF) to compress those pulses down to $\sim 5 \mathrm{fs}$. In addition to that, optical parametric amplifiers (OPAs) give the possibility to cover various spectral ranges and broad bandwidth amplification.

In order to generate isolated attosecond pulses with high photon energies via the process of HHG, one benefits from sub-cycle driving pulses to suppress multicycle emission of the harmonics. A long center wavelength increases the recollision energy between the electronic wavepacket and the parent ion. The scaling law of the HHG cutoff energy was reported as $\lambda^{2}$, while the efficiency drops with $\lambda^{\sim(-5)}$. Recently published sources of such kind are e.g. a combination of OPA and HCF, that amplify a spectrum at longer wavelength with subsequent compression $[1,2]$.

Our source presented here is exploiting the coherent waveform synthesis [3,4] of different OPAs to overcome the energy and bandwidth limitations of before mentioned sources. By controlling the carrier-envelope phase (CEP) and the relative phase among its channels it gives the possibility to create non-sinusoidal waveforms and to study how they can be exploited to control the HHG process [5].

\section{Parametric synthesis}

Our waveform synthesizer is pumped by a commercial cryogenically-cooled Ti:Sa amplifier delivering $20 \mathrm{~mJ}, 150 \mathrm{fs}$ pulses at $1 \mathrm{kHz}$ repetition rate. First, a small portion of the pulse

\footnotetext{
*e-mail: fabian.scheiba@cfel.de
} 
energy is used to pump a narrowband OPA that derives CEP stable pulses out of the non-CEP stabilized pump laser. Those pulses are then used to drive separate white-light generation (WLG) stages, each of them seeding a different spectral channel of the synthesizer [6]. Each channel consists of a three stage amplifier with final spectra shown in figure 1c) and 1d). At
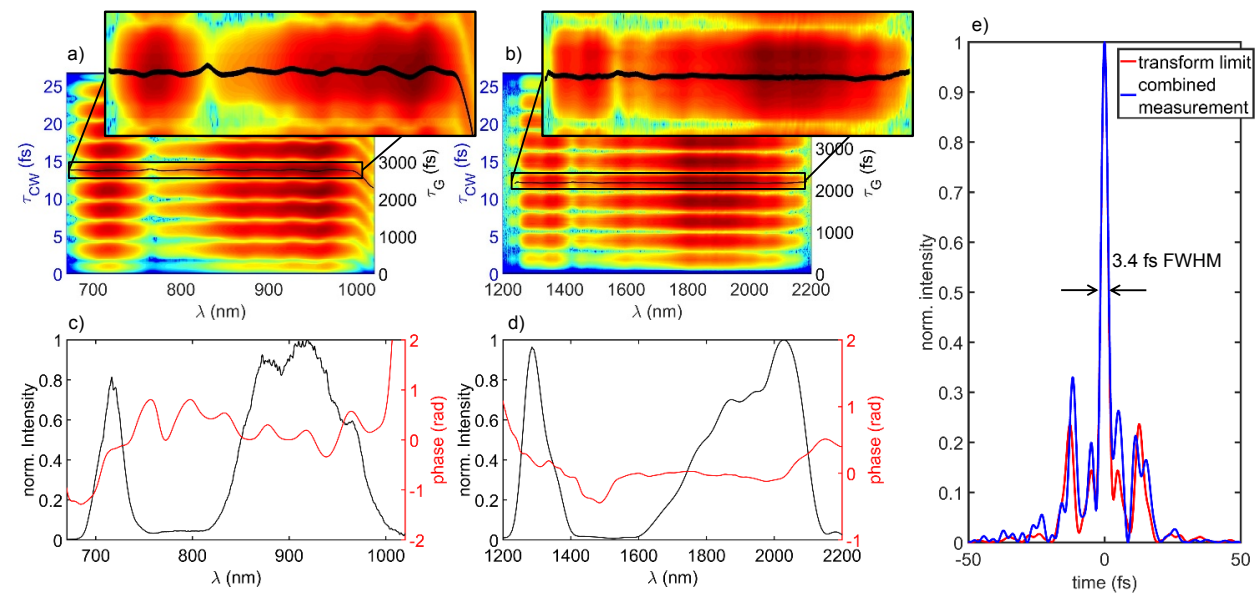

Figure 1. 2DSI traces of NIR (a) and IR (b) OPA with the cw delay of the ancillary pulse on the left $y$-axis and the retrieved group delay overlayed on the right $y$-axis. The spectra and spectral phases are shown in (c) and (d) resulting in $6.6 \mathrm{fs}$ and $7.2 \mathrm{fs}$ FWHM duration. The calculated FWHM of the synthesized pulse yields 3.4 fs (e) for perfect temporal overlap at time zero.

present, the near-infrared (NIR) and the infrared (IR) channels are in operation delivering respectively $100 \mu \mathrm{J}$ and $500 \mu \mathrm{J}$ energy (measured at the experimental interaction point) whereas a portion of $5 \mathrm{~mJ}$ from our driving laser is reserved for the planned third channel in the visible regime. The NIR and IR channels exploit the ultrabroadband phase-matching appearing at the degeneracy point, with signal wavelengt corresponding to double of the pump wavelengths $\left(\lambda_{P}=400 \mathrm{~nm}\right.$ and $\lambda_{P}=800 \mathrm{~nm}$ respectively), allowing for already broadband single channel spectra. The two channels can be independently optimized and compressed via a combination of bulk materials and custom designed double chirped mirrors [7]. The combination is obtained via custom designed dichroic mirrors. A temporal analysis has been performed using two-dimensional spectral shearing interferometry (2DSI) [8] for each channel (figure 1a) and 1b)) giving a FWHM of 6.6 fs for the NIR and 7.2 fs for the IR channel. By controlling the relative phase among the two channels it is possible to obtain a calculated overall pulse duration of $3.4 \mathrm{fs}$ FWHM, corresponding to 0.6 cycles at the central wavelength of $1.8 \mu \mathrm{m}$. The intensity of the synthesized waveform depending on the relative phase (or delay) among the two channels as shown in figure 2 a.

\section{Field control}

The sub-cycle duration of our pulses makes them very sensitive to any mechanical and environmental noise. We reduce the noise sources in the optical setup by using temperaturecontrolled breadboards, custom optics mounts and by minimizing the beam paths. Additionally an active control system enables further stabilization and control of the relative phase and CEP of the pulse via piezo actuators and single shot, every shot feedback electronics. The 
stability of the system was measured to a CEP noise of the frontend reading $\sim 200 \mathrm{mrad} \mathrm{rms}$ and the relative phase (RP) after the $3 \mathrm{rd}$ stage amplification of $\sim 100 \mathrm{mrad} \mathrm{rms}$. The energy fluctuations are well below $2 \%$ measured over 1 million shots for both channels.

\section{High Harmonic Generation}

We performed HHG in Argon first of all to test the capabilities of this source and proof its stability. The data were taken using a gas jet in a $\sim 1 \mathrm{~mm}$ glass capillary with a backing pressure of 300 mbar. The defined control over the electric field is greatly shown on the process of HHG when we control the incident electric field by varying the CEP or RP. The results are given in figure $2 \mathrm{~b}$ ). The yellow curve is the HH spectra from the NIR channel only while the blue and red curve show the spectra when NIR and IR are temporally and spatially overlapped in the gas jet. We observe a transition from the fringed spectrum to a continuum like shape which again depends on the RP between the channels. The rise of a
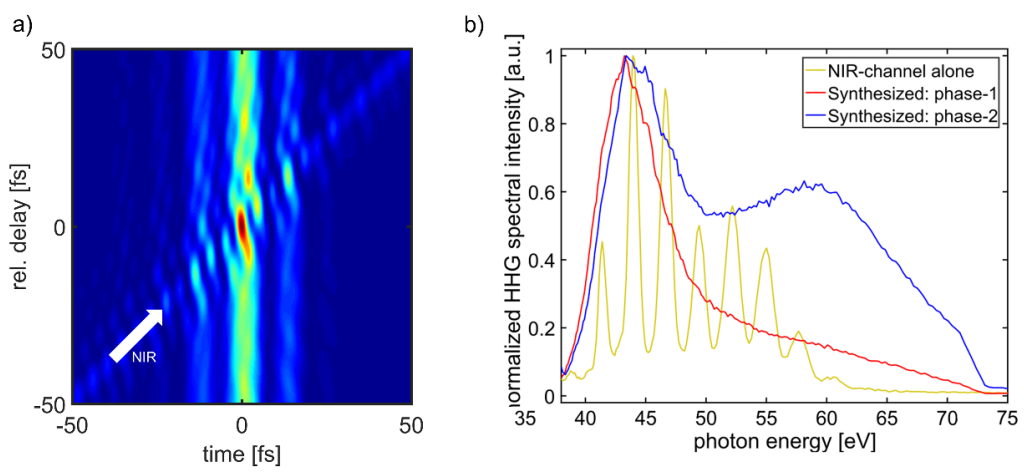

Figure 2. (a) Calculation of the temporal intensity when scanning the temporal overlap of IR and NIR channel. (b) HHG spectra of single NIR channel (yellow) and both channels with different relative phases (blue, red) indicating the transition from comb like harmonics to a single continuum depending on the temporal overlap of both pulses.

continuum for some defined settings of the RP is another indication of the subcycle nature of the synthesized pulse. We would like to point out that no gating technique was used for the production of the HHG spectra.

This work was supported by the Hamburg Centre for Ultrafast Imaging Structure, Dynamics and Control of Matter at the Atomic Scale" (CUI, DFG-EXC1074).

\section{References}

[1] T. Gaumnitz et al., Opt. Express 25, 27506-27518 (2017)

[2] F. J. Furch et al., Opt. Lett. 42, 2495-2498 (2017)

[3] O. D. Mücke et al., IEEE JSTQE 21, 1-12 (2015)

[4] A. Wirth et al., Science 334, 195-200 (2011)

[5] L. E. Chipperfield et al., Phys. Rev. Lett. 102, 063003 (2009)

[6] R. E. Mainz et al., CLEO Conf. Proc.g STu1I.6 (2016)

[7] S.-H. Chia et al., Optica 1, 315-322 (2014)

[8] J. R. Birge et al., Opt. Lett. 31, 2063-2065 (2006) 